\title{
A METHOD FOR GENERATING INFINITE POSITIVE SELF-ADJOINT TEST MATRICES AND RIESZ BASES*
}

\author{
C. V. M. VAN DER MEE ${ }^{\dagger}$ AND S. SEATZU ${ }^{\dagger}$ \\ Dedicated to Laura Gori on the occasion of her 70th birthday
}

\begin{abstract}
In this article we propose a method to easily generate infinite multi-index positive definite self-adjoint matrices as well as Riesz bases in suitable subspaces of $L^{2}\left(\mathbb{R}^{d}\right)$. The method is then applied to obtain some classes of multi-index Toeplitz matrices which are bounded and strictly positive on $\ell^{2}\left(\mathbb{Z}^{d}\right)$. The condition number of some of these matrices is also computed.
\end{abstract}

Key words. test matrix, nonuniform sampling, shift-invariant subspace, Riesz basis

AMS subject classifications. $42 \mathrm{C} 15,46 \mathrm{~A} 35$

DOI. $10.1137 / \mathrm{S} 0895479803432502$

1. Introduction. Matrices with special properties are important tools for testing numerical algorithms and software, while Riesz bases in different Hilbert spaces are important for solving many problems in approximation theory. However, whereas there are several methods for generating extensive classes of finite test matrices (see, e.g., $[16,11]$ ), we are not aware of methods for generating multi-index test matrices. Similarly, whereas there are methods for generating Riesz bases in subspaces of $L^{2}(\mathbb{R})$ and $L^{2}\left(\mathbb{R}^{+}\right)[13,18]$, we are not aware of general methods for generating Riesz bases in subspaces of $L^{2}\left(\mathbb{R}^{d}\right)$ for $d \geq 2$, except for grids of sampling points with, apart from a positive constant factor, only integer coordinates $[20,2]$. We note that there is an increasing interest in this topic both from the theoretical and the applicational points of view. Classes of multi-index positive definite test matrices could be used, in particular, to compare the effectiveness of preconditioning techniques in solving linear systems by the conjugate gradient method [22, 9, 23, 24].

In a recent joint paper [18] with Nashed on the sampling expansions of functions defined on the real line which belong to unitarily translation invariant reproducing kernel Hilbert spaces $H_{\phi}$, we have developed a method to generate both infinite positive self-adjoint matrices and Riesz bases in suitable subspaces of $H_{\phi}$. More precisely, starting from a real function $\phi \in L^{1}(\mathbb{R}) \cap L^{2}(\mathbb{R})$ whose Fourier transform $\hat{\phi}$ defined by $\hat{\phi}(\omega)=(2 \pi)^{-d / 2} \int_{\mathbb{R}^{d}} e^{i \omega \cdot x} \phi(x) d x$ does not vanish, we have represented the Hilbert space $H_{\phi}$ of all $f$ such that $(\hat{f} / \hat{\phi}) \in L^{2}(\mathbb{R})$ as a reproducing kernel Hilbert space with reproducing kernel

$$
k_{\phi}(t, u)=\kappa_{\phi}(t-u)=\int_{-\infty}^{\infty} \phi(x-t) \phi(x-u) d x .
$$

Assuming in addition that $\phi(\cdot)\left(1+(\cdot)^{2}\right)^{\gamma} \in L^{2}(\mathbb{R})$ for some $\gamma>1$, and taking a sequence of sampling points $\left\{t_{j}\right\}_{j=-\infty}^{\infty}$ such that $\left|t_{i}-t_{j}\right| \geq \varepsilon>0$ for $i \neq j$, it has been

\footnotetext{
${ }^{*}$ Received by the editors July 8, 2003; accepted for publication (in revised form) by H. J. Woerdeman August 27, 2004; published electronically May 6, 2005. The research leading to this article was supported in part by MIUR under the COFIN grant 2002014121 and by INdAM-GNCS.

http://www.siam.org/journals/simax/26-4/43250.html

${ }^{\dagger}$ Dipartimento di Matematica e Informatica, Università di Cagliari, viale Merello 92, 09123 Cagliari, Italy (cornelis@bugs.unica.it,seatzu@unica.it).
} 
proved that for all $f$ in a suitable closed subspace $\mathcal{X}_{\phi}$ of $H_{\phi}$ we have the following results:

(a) The Gram matrix

$$
G_{i j}=k_{\phi}\left(t_{i}, t_{j}\right), \quad i, j \in \mathbb{Z},
$$

is bounded and strictly positive self-adjoint on $\ell^{2}(\mathbb{Z})$.

(b) The sequence

$$
\left\{k_{\phi}\left(\cdot, t_{j}\right)\right\}_{j=-\infty}^{\infty}
$$

is a Riesz basis in $\mathcal{X}_{\phi}$.

(c) The sampling expansion

$$
f(t)=\frac{1}{\|\phi\|_{2}^{2}} \sum_{j=-\infty}^{\infty} f\left(t_{j}\right) \kappa_{\phi}\left(t-t_{j}\right), \quad t \in \mathbb{R}
$$

is valid for every $f \in \mathcal{X}_{\phi}$. Note that $\kappa_{\phi}(0)=\|\phi\|_{2}^{2}$.

Though the closure $\mathcal{X}_{\phi}$ of the linear span of the functions $\left\{k_{\phi}\left(\cdot, t_{j}\right)\right\}_{j=-\infty}^{\infty}$ has not been explicitly specified, in [18] various examples have been worked out in detail.

In [18] the main emphasis of the research has been on the development of sampling expansions in unitarily translation invariant reproducing kernel Hilbert spaces. Although in the present article we have generalized the main results in [18] on sampling expansions for functions on the line to sampling expansions for functions on $\mathbb{R}^{d}$, the present authors are primarily interested in the multi-index Toeplitz matrices arising as Gram matrices of the Riesz bases involved in the case of equidistant sampling points. These matrices are presently being used as test matrices in the development of numerical methods for solving multi-index Toeplitz systems. We are, in particular, interested in comparing the effectiveness of recent preconditioning techniques in solving linear systems by the conjugate gradient method with the most commonly used preconditioning techniques. We are also interested in solving large multi-index Toeplitz systems by using the solution of the corresponding infinite Toeplitz system. For these reasons the present paper contains many explicit examples whose entries have Gaussian, exponential, or algebraic decay away from the diagonal, including the condition numbers of some of the Toeplitz matrices generated.

The outline of the paper is as follows. In section 2 we compile some useful definitions and results involving Gram matrices, Riesz bases, and frame inequalities. In section 3 we illustrate the method proposed for generating positive definite multiindex Toeplitz matrices. In section 4 we present various examples of strictly positive self-adjoint multi-index Toeplitz matrices. Finally, in Appendix A we present a duplication formula for Bessel polynomials that has been used to generate a specific class of multi-index Toeplitz matrices, while in Appendix B we compute the condition numbers of some of the Toeplitz matrices introduced.

Throughout this article, $|\cdot|$ will stand for the Euclidean vector norm or the absolute value of a real or complex number.

2. Preliminaries. Given a complex Hilbert space $H$, a sequence $\left\{f_{n}\right\}_{n \in J}, J \subseteq$ $\mathbb{Z}^{d}$ and $J$ infinite, of vectors in $H$ is called a frame (cf. $[10,26]$ ) if there exist positive constants $C_{1}, C_{2}$ such that

$$
C_{1}\|f\|_{H} \leq\left[\sum_{n \in J}\left|\left\langle f, f_{n}\right\rangle_{H}\right|^{2}\right]^{1 / 2} \leq C_{2}\|f\|_{H}, \quad f \in H .
$$


These inequalities are called the frame inequalities. The frame is called an exact frame if the removal of any vector from the frame causes it not to be a frame anymore. Given a frame, the linear operator $T$ defined by $T f=\sum_{n \in J}\left\langle f, f_{n}\right\rangle_{H} f_{n}$ is a bounded linear operator on $H$. Further, if $\left\{f_{n}\right\}_{n \in J}$ is an exact frame, for every $f \in H$ there exists a unique sequence $\left\{a_{n}\right\}_{n \in J}$ such that

$$
f=\sum_{n \in J} a_{n} f_{n}
$$

where $\sum_{n \in J}\left|a_{n}\right|^{2}<\infty$. A well-known result $[10,26]$ states that a sequence $\left\{f_{n}\right\}_{n \in J}$ in a separable Hilbert space $H$ is an exact frame if and only if it is a Riesz basis in $H$ (i.e., if it can be obtained from an orthonormal basis in $H$ by applying a boundedly invertible operator).

Proposition 2.1. Let $H$ be a complex Hilbert space and let $\left\{f_{j}\right\}_{j \in J}, J \subseteq \mathbb{Z}^{d}$, be a sequence of functions in $H$. Then the following statements are equivalent:

1. There exist positive constants $C_{1}, C_{2}$ such that

$$
C_{1}\|f\|_{H} \leq\left[\sum_{j \in J}\left|\left\langle f, f_{j}\right\rangle_{H}\right|^{2}\right]^{1 / 2} \leq C_{2}\|f\|_{H}, \quad f \in H,
$$

holds for every $f \in H$ and no such relation holds for any proper subset of functions $\left\{f_{j}\right\}$.

2. The sequence $\left\{f_{j}\right\}_{j \in J}$ is a Riesz basis in $H$.

3. The sequence of functions $\left\{f_{j}\right\}_{j \in J}$ is complete, and the Gram matrix $G_{i j}=$ $\left(\left\langle f_{i}, f_{j}\right\rangle_{H}\right)_{i, j \in J}$ is bounded and strictly positive self-adjoint on $\ell^{2}(J)$.

Recall that by a reproducing kernel Hilbert space of functions supported on a set $S$ we mean a (complex) Hilbert space of functions on $S$, where all of the evaluation functionals $\xi_{t}(f)=f(t)$, for $f \in H$ and each fixed $t \in S$, are continuous [3, 5, 17]. Then, by the Riesz representation theorem, for each $t \in S$ there exists a unique element $k_{t} \in H$ such that

$$
f(t)=\left\langle f, k_{t}\right\rangle, \quad f \in H,
$$

where $\langle\cdot, \cdot\rangle$ is the scalar product on $H$. We then call $k(t, u)=\left\langle k_{t}, k_{u}\right\rangle$, for $t, u \in S$, the reproducing kernel of $H$. Clearly, $k(\cdot, \cdot)$ is Hermitian and positive definite.

In [18], Proposition 2.1 has been applied more specifically to the situation in which $H$ is a reproducing kernel Hilbert space of complex-valued functions on a set $S$ with reproducing kernel $k(t, s)$ and $f_{j}(t)=k\left(t, t_{j}\right) / \sqrt{k\left(t_{j}, t_{j}\right)}$ for a sequence of points $\left\{t_{j}\right\}_{j \in J}$ in $S$. Then, under any of the conditions of Proposition 2.1, for every $f \in H$ we have the moment expansion

$$
f(t)=\sum_{j \in J}\left\langle f, f_{j}\right\rangle_{H} f_{j}(t)
$$

When $J=\mathbb{Z}^{d}$, the Gram matrix $\left\{G_{i j}\right\}_{i, j \in \mathbb{Z}^{d}}$ is a multi-index Toeplitz matrix (i.e., $G_{i j}=G_{i-j}$ for $\left.i, j \in \mathbb{Z}^{d}\right)$.

The following elementary result has been adapted from [18].

Proposition 2.2. Let $J=\mathbb{Z}^{d}$. Then the statements of Proposition 2.1 and the following two claims are equivalent: 
1. The sequence of functions $\left\{f_{j}\right\}_{j \in \mathbb{Z}^{d}}$ is complete, and the multi-index Toeplitz matrix $\left(G_{i-j}\right)_{i, j \in \mathbb{Z}^{d}}$ defined by

$$
G_{i-j}=\left\langle f_{i}, f_{j}\right\rangle_{H}
$$

is bounded and strictly positive self-adjoint on $\ell^{2}\left(\mathbb{Z}^{d}\right)$.

2. The sequence of functions $\left\{f_{j}\right\}_{j \in \mathbb{Z}^{d}}$ is complete, and the symbol

$$
\hat{G}(s)=\sum_{j \in \mathbb{Z}^{d}} s^{j} G_{j}, \quad s=\left(s_{1}, \ldots, s_{d}\right), \quad\left|s_{1}\right|=\cdots=\left|s_{d}\right|=1,
$$

is positive, essentially bounded, and essentially bounded away from zero.

If any of these conditions holds and $J=\mathbb{Z}^{d}$, the condition number of $G$ equals

$$
\frac{\max _{s \in \mathbb{T}^{d}} \hat{G}(s)}{\min _{s \in \mathbb{T}^{d}} \hat{G}(s)},
$$

where $\mathbb{T}^{d}$ is the d-dimensional torus.

3. The method. Let $\phi$ be a real function in $L^{1}\left(\mathbb{R}^{d}\right) \cap L^{2}\left(\mathbb{R}^{d}\right)$ and let

$$
k_{\phi}(t, u)=\kappa_{\phi}(t-u)=\int_{\mathbb{R}^{d}} \phi(x-t) \phi(x-u) d x=\int_{\mathbb{R}^{d}} e^{-i \omega(t-u)}|\hat{\phi}(\omega)|^{2} d \omega .
$$

Then

$$
\hat{\kappa}_{\phi}(\omega)=(2 \pi)^{d / 2}|\hat{\phi}(\omega)|^{2},
$$

where $\hat{\phi}(\omega)=(2 \pi)^{-d / 2} \int_{\mathbb{R}^{d}} e^{i \omega \cdot x} \phi(x) d x$. Now suppose $\left\{t_{j}\right\}_{j \in J}, J \subset \mathbb{Z}^{d}$, is an infinite sequence of sampling points in $\mathbb{R}^{d}$ and

$$
\left(G_{\phi}\right)_{i j}:=k_{\phi}\left(t_{i}, t_{j}\right)=\int_{\mathbb{R}^{d}} \phi\left(x-t_{i}\right) \phi\left(x-t_{j}\right) d x, \quad i, j \in J,
$$

the associated Gram matrix. When the sampling points are equidistant (i.e., when $t_{j}=\alpha j$ for some $\left.\alpha>0\right), G_{\phi}$ is a multi-index Toeplitz matrix whose symbol we define by

$$
\hat{G}(s, \alpha)=\sum_{j \in \mathbb{Z}^{d}} s^{j} \int_{\mathbb{R}^{d}} \phi(x) \phi(x+\alpha j) d x=\sum_{j \in \mathbb{Z}^{d}} s^{j} \kappa_{\phi}(\alpha j),
$$

where the series converge uniformly and absolutely in $s$ on the $d$-dimensional torus $\mathbb{T}^{d}$ if the condition

$$
\sum_{j \in \mathbb{Z}^{d}}\left|\kappa_{\phi}(\alpha j)\right|<\infty
$$

is satisfied.

The condition that $\hat{\phi}(\omega) \neq 0$ for every $\omega \in \mathbb{R}^{d}$ is sufficient for $k_{\phi}(\cdot, \cdot)$ to be a reproducing kernel on $S=\mathbb{R}^{d}$. Indeed, let $t_{1}, \ldots, t_{n}$ be distinct points in $\mathbb{R}^{d}$. Then for every nontrivial $n$-tuple $\left(\xi_{1}, \ldots, \xi_{n}\right)$ of complex numbers we have

$$
\begin{aligned}
\sum_{i, j=1}^{n} k_{\phi}\left(t_{i}, t_{j}\right) \xi_{i} \overline{\xi_{j}} & =\int_{\mathbb{R}^{d}}|\hat{\phi}(\omega)|^{2} \sum_{i, j=1}^{n} e^{i\left(t_{i}-t_{j}\right) \cdot \omega} \xi_{i} \overline{\xi_{j}} d \omega \\
& =\int_{\mathbb{R}^{d}}|\hat{\phi}(\omega)|^{2}\left|\sum_{i=1}^{n} e^{i t_{i} \cdot \omega} \xi_{i}\right|^{2} d \omega>0,
\end{aligned}
$$


which proves that $k_{\phi}(\cdot, \cdot)$ is a reproducing kernel on $S=\mathbb{R}^{d}$ if $\hat{\phi}(\omega) \neq 0$ for every $\omega \in \mathbb{R}^{d}$. As in [18], we now easily identify the corresponding reproducing kernel Hilbert space $H_{\phi}$ (cf. $[3,5,17]$ for reproducing kernel Hilbert spaces) with the complex Hilbert space of all measurable functions $f$ on $\mathbb{R}^{d}$ such that $(\hat{f} / \hat{\phi}) \in L^{2}\left(\mathbb{R}^{d}\right)$, endowed with the norm

$$
\|f\|_{H_{\phi}}=\frac{1}{(2 \pi)^{d / 2}}\left[\int_{\mathbb{R}^{d}}|\hat{f}(\omega)|^{2} \frac{d \omega}{|\hat{\phi}(\omega)|^{2}}\right]^{1 / 2} .
$$

The following result provides a general condition on $\phi$ and the sampling points in order that the Gram matrix $\left\{\kappa_{\phi}\left(t_{i}, t_{j}\right)\right\}_{i, j \in J}$ be bounded on $\ell^{2}(J)$. In the case of equidistant sampling points, we actually prove that condition (3.3) holds. Note that all of the examples given in the next section satisfy these conditions.

THEOREM 3.1. Let the distinct sampling points $\left\{t_{j}\right\}_{j \in J}$, with $J \subseteq \mathbb{Z}^{d}$ an infinite set, satisfy $\left|t_{i}-t_{j}\right| \geq \varepsilon>0$ for $i \neq j$ in $J$. Further, let $\phi$ have the property

$$
\exists \gamma>d: \int_{\mathbb{R}^{d}}\left(1+|x|^{2}\right)^{\gamma} \phi(x)^{2} d x<\infty .
$$

Then the Gram matrix $\left\{k_{\phi}\left(t_{i}, t_{j}\right)\right\}_{i, j \in J}$ is bounded on $\ell^{2}(J)$. In particular, if $t_{i}=\alpha i$ $\left(i \in J=\mathbb{Z}^{d}\right)$ for some $\alpha>0$, then (3.3) is satisfied.

Proof. Note that

$$
\sup _{i \in J} \sum_{j \in J}\left|k_{\phi}\left(t_{i}, t_{j}\right)\right|=\sup _{i \in J} \sum_{j \in J}\left|\kappa_{\phi}\left(t_{i}-t_{j}\right)\right|
$$

is an upper bound for the norm of the Gram matrix on $\ell^{2}(J)$. Therefore,

$$
\begin{aligned}
(1+|t|)^{\gamma}\left|\kappa_{\phi}(t)\right| & \leq \int_{\mathbb{R}^{d}}(1+|x|)^{\gamma}|\phi(x)| \cdot(1+|x+t|)^{\gamma}|\phi(x+t)| d x \\
& \leq \int_{\mathbb{R}^{d}}(1+|x|)^{2 \gamma} \phi(x)^{2} d x \leq 2^{\gamma} \int_{\mathbb{R}^{d}}\left(1+|x|^{2}\right)^{\gamma} \phi(x)^{2} d x,
\end{aligned}
$$

which implies that (3.5) is bounded above when $\left|t_{i}-t_{j}\right| \geq \varepsilon$ for $i \neq j$.

We now give sufficient conditions on $\phi$ and the sampling points for the Gram matrix $\left\{\kappa_{\phi}\left(t_{i}, t_{j}\right)\right\}_{i, j \in J}$ to be bounded below on $\ell^{2}(J)$ by a positive multiple of the identity. With Theorem 3.1, we then obtain sufficient conditions on $\phi$ and the sampling points in order that this Gram matrix be bounded and strictly positive self-adjoint on $\ell^{2}(J)$ and that the frame inequalities (2.1) be satisfied. All of the examples of the next section satisfy these conditions. The two proofs we give are based in part on ideas of Schaback [21, Theorem 3.1].

TheOREM 3.2. Let $\left(t_{j}\right)_{j \in \mathbb{Z}^{d}}$ be sampling points with $t_{0}=0$ and

$$
\left|t_{i s}-t_{j s}\right| \geq \varepsilon\left|i_{s}-j_{s}\right|>0, \quad i, j \in \mathbb{Z}^{d} \text { with } t_{i s} \neq t_{j s} .
$$

Let $\phi$ be a real function in $L^{1}\left(\mathbb{R}^{d}\right) \cap L^{2}\left(\mathbb{R}^{d}\right)$ satisfying the conditions of Theorem 3.1 whose Fourier transform $\hat{\phi}(\omega) \neq 0$ for $\max \left(\left|\omega_{1}\right|, \ldots,\left|\omega_{d}\right|\right) \leq M$ for any $M>$ $\pi / \varepsilon \sqrt{3\left(2^{1 / d}-1\right)}$. Then the Gram matrix $\left\{\kappa_{\phi}\left(t_{i}-t_{j}\right)\right\}_{i, j \in \mathbb{Z}^{d}}$ is bounded and strictly positive self-adjoint on $\ell^{2}\left(\mathbb{Z}^{d}\right)$. 
Moreover, if $\hat{\phi}(\omega) \neq 0$ for all $\omega \in \mathbb{R}^{d}$ and $\mathcal{X}_{\phi}$ denotes the closed linear span of $\kappa_{\phi_{j}}(x)=\kappa_{\phi}\left(x-t_{j}\right), j \in \mathbb{Z}^{d}$ in $H_{\phi}$, then there exist positive constants $C_{1}, C_{2}$ such that the frame inequalities

$$
C_{1}\|f\|_{H_{\phi}} \leq\left[\sum_{j \in \mathbb{Z}^{d}}\left|f\left(t_{j}\right)\right|^{2}\right]^{1 / 2} \leq C_{2}\|f\|_{H_{\phi}}, \quad f \in \mathcal{X}_{\phi}
$$

hold. Consequently, $\left\{\kappa_{\phi_{j}}\right\}_{j \in \mathbb{Z}^{d}}$ is a Riesz basis in $\mathcal{X}_{\phi}$ and for each $f \in \mathcal{X}_{\phi}$ we have the interpolating expansion

$$
f(t)=\frac{1}{\|\phi\|_{2}^{2}} \sum_{j \in \mathbb{Z}^{d}} f\left(t_{j}\right) \kappa_{\phi}\left(t-t_{j}\right) .
$$

Proof. We present two proofs, the first one adapted to $\phi$ such that $\hat{\phi}(\omega)$ is zero free for $\max \left(\left|\omega_{1}\right|, \ldots,\left|\omega_{d}\right|\right) \leq 2 M$, and the second one adapted to $\phi$ such that $\hat{\phi}(\omega)$ is zero free for $|\omega| \leq 2 R$, where $M$ and $R$ are specified in the first and second proofs, respectively.

First proof. For $N \in \mathbb{N}$ and any set of $N$ sampling points and arbitrary complex numbers $c_{1}, \ldots, c_{N}$, by Parseval's theorem we have

$$
\begin{aligned}
& \sum_{j, r=1}^{N} c_{j} \overline{c_{r}} \kappa_{\phi}\left(t_{j}-t_{r}\right)=\int_{\mathbb{R}^{d}}\left|\sum_{j=1}^{N} c_{j} \phi\left(x-t_{j}\right)\right|^{2} d x=\int_{\mathbb{R}^{d}}\left|\sum_{j=1}^{N} c_{j} e^{i \omega \cdot t_{j}} \hat{\phi}(\omega)\right|^{2} d \omega \\
& \quad \geq\left(\inf _{\left|\omega_{s}\right| \leq 2 M, s=1, \ldots, d}|\hat{\phi}(\omega)|^{2}\right) \int_{-2 M}^{2 M} \cdots \int_{-2 M}^{2 M} \Psi_{M}(\omega) \sum_{i, j=1}^{N} c_{i} \overline{c_{j}} e^{i \omega \cdot\left(t_{i}-t_{j}\right)} d \omega,
\end{aligned}
$$

where

$$
\Psi_{M}(\omega)= \begin{cases}(2 M)^{-d} \prod_{s=1}^{d}\left(2 M-\left|\omega_{s}\right|\right), & \left|\omega_{s}\right| \leq 2 M, s=1, \ldots, d \\ 0 & \text { otherwise }\end{cases}
$$

Putting

$$
B(u)=\int_{-1}^{1}(1-|\zeta|) e^{i \zeta u} d \zeta= \begin{cases}\left(\frac{\sin \left(\frac{1}{2} u\right)}{\frac{1}{2} u}\right)^{2}, & u \neq 0 \\ 1, & u=0\end{cases}
$$

we obtain for $t_{j}=\left(t_{j 1}, \ldots, t_{j d}\right)(j=1, \ldots, N)$

$$
\int_{\mathbb{R}^{d}}\left|\sum_{j=1}^{N} c_{j} \phi\left(x-t_{j}\right)\right|^{2} d x \geq\left(\inf _{\left|\omega_{s}\right| \leq 2 M, s=1, \ldots, d}|\hat{\phi}(\omega)|^{2}\right) \sum_{i, j=1}^{N} c_{i} \overline{c_{j}} A_{i j},
$$

where

$$
A_{i j}=\prod_{s=1}^{d} B\left(2 M\left(t_{i s}-t_{j s}\right)\right)
$$


Now choose $\varepsilon>0$ such that $\left|t_{i s}-t_{j s}\right| \geq \varepsilon\left|i_{s}-j_{s}\right|$ for $t_{i s} \neq t_{j s}$. Then in view of

$$
\begin{aligned}
0 & <\prod_{s=1}^{d} B\left(2 M\left(t_{i s}-t_{j s}\right)\right)=\prod_{\substack{s=1 \\
t_{i s} \neq t_{j s}}}^{d} B\left(2 M\left(t_{i s}-t_{j s}\right)\right) \\
& \leq \prod_{\substack{s=1, \ldots, d \\
t_{i s} \neq t_{j s}}} \frac{1}{\left(M \varepsilon\left(i_{s}-j_{s}\right)\right)^{2}}=\prod_{\substack{s=1, \ldots, d \\
i_{s} \neq j_{s}}} \frac{1}{\left(M \varepsilon\left(i_{s}-j_{s}\right)\right)^{2}} .
\end{aligned}
$$

We easily prove, by induction on $d$, that

$$
\begin{aligned}
\sum_{j \in \mathbb{Z}^{d}} \prod_{s=1}^{d} B\left(2 M\left(t_{i s}-t_{j s}\right)\right) & \leq 1+\sum_{\substack{j \in \mathbb{Z}^{d} \\
j \neq i}} \prod_{s=1}^{d} B\left(2 M\left(t_{i s}-t_{j s}\right)\right) \\
& \leq 1+\sum_{\substack{j \in \mathbb{Z}^{d} \\
j \neq i}} \prod_{\substack{s=1, \ldots, d \\
i_{s} \neq j_{s}}} \frac{1}{\left(M \varepsilon\left(i_{s}-j_{s}\right)\right)^{2}} \\
& \leq 1+\sum_{\substack { j \in \mathbb{Z}^{d} \backslash\{0\} \\
\begin{subarray}{c}{s=1, \ldots, d \\
j_{s} \neq 0{ j \in \mathbb { Z } ^ { d } \backslash \{ 0 \} \\
\begin{subarray} { c } { s = 1 , \ldots , d \\
j _ { s } \neq 0 } }\end{subarray}} \frac{1}{\left(M \varepsilon j_{s}\right)^{2}} \leq(1+2 S(M \varepsilon))^{d},
\end{aligned}
$$

where

$$
S(z)=\sum_{i=1}^{\infty} \frac{1}{(z i)^{2}}=\frac{\pi^{2}}{6 z^{2}}
$$

Using Gershgorin's theorem [12, Theorem 8.1.3], it appears that the real symmetric matrix $\left(A_{i j}\right)_{i, j=1}^{N}$ with elements defined by the right-hand side of (3.10) has all of its diagonal elements equal to 1 , and hence all of its eigenvalues $\lambda$ are real and satisfy

$$
|1-\lambda| \leq \max _{i=1, \ldots, N} \sum_{\substack{j=1 \\ j \neq i}}^{N}\left|A_{i j}\right| .
$$

Thus its eigenvalues can be found in the open interval from $2-(1+2 S(M \varepsilon))^{d}$ to $(1+$ $2 S(M \varepsilon))^{d}$ whose endpoints do not depend on $N$. Thus if $M>\pi /\left(\varepsilon \sqrt{3}\left(2^{1 / d}-1\right)^{1 / 2}\right)$, this matrix is positive definite. Therefore, for this choice of $M$ the lower bound (3.8) extends to arbitrary subsets of the set of the sampling points, and hence the Gram matrix $\left\{\kappa_{\phi}\left(t_{i}-t_{j}\right)\right\}_{i, j \in \mathbb{Z}}$ is strictly positive self-adjoint. Its boundedness follows from Theorem 3.1. The frame inequalities (3.6) now follow with the help of Proposition 2.1. Finally, (3.7) is immediate from (2.2), (3.6), and $k_{\phi}\left(t_{j}, t_{j}\right)=\kappa_{\phi}(0)=\|\phi\|_{2}^{2}$.

Second proof. Let $R$ be a positive real number and let $\chi_{R}^{d}(x)$ be the characteristic function of the sphere in $\mathbb{R}^{d}$ with center the origin and radius $R$. Then

$$
0 \leq \int_{\mathbb{R}^{d}} d x \chi_{R}^{d}(x-t) \chi_{R}^{d}(x-s) \leq R^{d} V_{d}, \quad t, s \in \mathbb{R}^{d},
$$

where $V_{d}$ is the volume of the unit ball in $\mathbb{R}^{d}$. Then for $N \in \mathbb{N}$ and any set of $N$ 
sampling points and arbitrary complex numbers $c_{1}, \ldots, c_{N}$, we have

$$
\begin{aligned}
\sum_{j, r=1}^{N} c_{j} \overline{c_{r}} \kappa_{\phi}\left(t_{j}-t_{r}\right) & =\int_{\mathbb{R}^{d}}\left|\sum_{j=1}^{N} c_{j} \phi\left(x-t_{j}\right)\right|^{2} d x=\int_{\mathbb{R}^{d}}\left|\sum_{j=1}^{N} c_{j} e^{i \omega \cdot t_{j}} \hat{\phi}(\omega)\right|^{2} d \omega \\
& \geq\left(\inf _{|\omega| \leq 2 R}|\hat{\phi}(\omega)|^{2}\right) \int_{|\omega| \leq 2 R} \Psi_{R}^{d}(\omega) \sum_{i, j=1}^{N} c_{i} \overline{c_{j}} e^{i \omega \cdot\left(t_{i}-t_{j}\right)} d \omega \\
& \geq\left(\inf _{|\omega| \leq 2 R}|\hat{\phi}(\omega)|^{2}\right) \sum_{i, j=1}^{N} c_{i} \overline{c_{j}} F_{d}\left(R, t_{i}-t_{j}\right),
\end{aligned}
$$

where $\hat{\phi}(\omega)$ is zero free for $|\omega| \leq 2 R$,

$$
\Psi_{R}^{d}(\omega)=\frac{1}{R^{d} V_{d}} \int_{\mathbb{R}^{d}} \chi_{R}^{d}(\xi) \chi_{R}^{d}(\xi-\omega) d \xi
$$

and

$$
F_{d}(R, t)=\int_{|\omega| \leq 2 R} \Psi_{R}^{d}(\omega) e^{i \omega \cdot t} d \omega=(2 \pi)^{d / 2} \hat{\Psi}_{R}^{d}(t)=\frac{(2 \pi)^{d}}{R^{d} V_{d}}\left|\hat{\chi}_{R}^{d}(t)\right|^{2} .
$$

Using $[14,8.411(5)$ and $6.561(5)]$ and $S_{d-2}=2 \pi^{(d-1) / 2} / \Gamma((d-1) / 2)$ we easily compute

$$
\begin{aligned}
\hat{\chi}_{R}^{d}(t) & =(2 \pi)^{-d / 2} S_{d-2} \int_{0}^{R} d r r^{d-1} \int_{0}^{\pi} d \varphi_{1}\left(\sin \varphi_{1}\right)^{d-2} e^{i r|t| \cos \varphi_{1}} \\
& =(2 \pi)^{-d / 2} R^{d} \int_{0}^{1} d \rho \rho^{d-1} \int_{0}^{\pi}\left(\sin \varphi_{1}\right)^{d-2} \cos \left(\rho R|t| \cos \varphi_{1}\right) \\
& =R^{2}\left(\frac{R}{|t|}\right)^{\frac{d-2}{2}} \int_{0}^{1} d \rho \rho^{\frac{d}{2}} J_{\frac{d-2}{2}}(\rho R|t|)=\left(\frac{R}{|t|}\right)^{\frac{d}{2}} J_{\frac{d}{2}}(R|t|),
\end{aligned}
$$

so that

$$
F_{d}(R, t)=\frac{(2 \pi)^{d}}{V_{d}|t|^{d}} J_{\frac{d}{2}}(R|t|)^{2} \text { and hence } F_{d}(R, 0)=\frac{(\pi R)^{d}}{V_{d} \Gamma\left(\frac{d+2}{2}\right)^{2}} .
$$

According to $[14,8.479]$, for $|t| \geq(d / 2 R)$ we have the estimate

$$
F_{d}(R, t)=\frac{(2 \pi)^{d}}{V_{d}|t|^{d}} J_{\frac{d}{2}}(R|t|)^{2} \leq \frac{2}{\pi} \frac{(2 \pi)^{d}}{V_{d}|t|^{d}} \frac{1}{\sqrt{(R|t|)^{2}-\left(\frac{d}{2}\right)^{2}}},
$$

and hence for $|t| \geq((\mu d) /(2 R))$ with fixed $\mu>1$ we have

$$
F_{d}(R, t) \leq \frac{1}{\pi V_{d} R \sqrt{\mu^{2}-1}}\left(\frac{2 \pi}{|t|}\right)^{d+1} .
$$

Now choose $\varepsilon>0$ such that $\left|t_{i}-t_{j}\right| \geq \varepsilon\left[\sum_{s=1}^{d}\left(i_{s}-j_{s}\right)^{2}\right]^{1 / 2}$. Then for $\varepsilon \geq$ $((\mu d) /(2 R))$ and some $\mu>1$ we have for $i \neq j$

$$
F_{d}\left(R, t_{i}-t_{j}\right) \leq \frac{(2 \pi / \varepsilon)^{d+1}}{\pi V_{d} R \sqrt{\mu^{2}-1}} \frac{1}{\left[\sum_{s=1}^{d}\left(i_{s}-j_{s}\right)^{2}\right]^{\frac{d+1}{2}}} .
$$


Therefore, for $|t| \geq((\mu d) /(2 R))$ with fixed $\mu>1$ we have

$$
\max _{i=1, \ldots, N} \sum_{\substack{j=1 \\ j \neq i}}^{N} F_{d}\left(R, t_{i}-t_{j}\right) \leq \frac{(2 \pi / \varepsilon)^{d+1}}{\pi V_{d} R \sqrt{\mu^{2}-1}} S_{[d]},
$$

where $S_{[d]}=\sum_{0 \neq j \in \mathbb{Z}^{d}}\left[j_{1}^{2}+\cdots+j_{d}^{2}\right]^{-\frac{d+1}{2}}$. Using Gershgorin's theorem [12, Theorem 8.1.3], it appears that the real symmetric matrix $\left(F\left(t_{i}-t_{j}\right)\right)_{i, j=1}^{N}$, appearing in (3.11), has its eigenvalues in the open interval of half-length $(2 \pi / \varepsilon)^{d+1} S_{[d]} / \pi V_{d} R \sqrt{\mu^{2}-1}$ centered about $F_{d}(R, 0)$. Consequently, if $\varepsilon$ strictly exceeds the number $\varepsilon_{0}(R, d, \mu)$ defined by

$$
\varepsilon_{0}(R, d, \mu)=\max \left(\frac{\mu d}{2 R}, \frac{2}{R}\left[\frac{S_{[d]} \Gamma\left(\frac{d+2}{2}\right)^{2}}{\sqrt{\mu^{2}-1}}\right]^{\frac{1}{d+1}}\right)
$$

for some $\mu>1$, then the real symmetric matrix $\left(F\left(t_{i}-t_{j}\right)\right)_{i, j=1}^{N}$ appearing in (3.11) is positive definite, irrespective of the choice of finite subset of the sampling points. The frame inequalities (3.6) now follow with the help of Proposition 2.1. Finally, (3.7) is immediate from (2.2), (3.6), and $k_{\phi}\left(t_{j}, t_{j}\right)=\kappa_{\phi}(0)=\|\phi\|_{2}^{2}$.

Assuming $\hat{\phi}(\omega) \neq 0$ for all $\omega \in \mathbb{R}^{d}$ and given the finite linear combination $\sum_{j} c_{j} \kappa_{\phi}\left(\cdot-t_{j}\right)$ in $\mathcal{X}_{\phi}$, we easily compute that

$$
\begin{aligned}
\left\|\sum_{j} c_{j} \kappa_{\phi}\left(\cdot-t_{j}\right)\right\|_{H_{\phi}}^{2} & =\frac{1}{(2 \pi)^{d}} \int_{\mathbb{R}^{d}}\left|\sum_{j} c_{j} e^{i \omega \cdot t_{j}}\right|^{2}\left|\hat{\kappa}_{\phi}(\omega)\right|^{2} \frac{d \omega}{|\hat{\phi}(\omega)|^{2}} \\
& =\int_{\mathbb{R}^{d}}\left|\sum_{j} c_{j} e^{i \omega \cdot t_{j}}\right|^{2}|\hat{\phi}(\omega)|^{2} d \omega .
\end{aligned}
$$

Hence if $\boldsymbol{t}=\left\{t_{j}: j \in \mathbb{Z}^{d}\right\}$ denotes the set of sampling points, then the image $\mathcal{F}\left[\mathcal{X}_{\phi}\right]$ of $\mathcal{X}_{\phi}$ under the Fourier transformation $\mathcal{F}$ coincides with the completion $\mathcal{A P}_{\boldsymbol{t}, \phi}$ of the vector space of $d$-variate almost periodic polynomials with spectrum within $\boldsymbol{t}$ with respect to the scalar product

$$
(f, g)_{\mathcal{X}_{\phi}}=\int_{\mathbb{R}^{d}} f(\omega) \overline{g(\omega)}|\hat{\phi}(\omega)|^{2} d \omega .
$$

Here by the spectrum of a $d$-variate almost periodic function $f: \mathbb{R}^{d} \rightarrow \mathbb{C}$ we mean the set of all $t \in \mathbb{R}^{n}$ for which $\lim _{T \rightarrow+\infty} \frac{1}{T^{d}} \int_{0}^{T} \cdots \int_{0}^{T} e^{-i x \cdot t} f(x) d x \neq 0$, where we note (cf. [6]) that

$$
\lim _{T \rightarrow+\infty} \frac{1}{T^{d}} \int_{0}^{T} \cdots \int_{0}^{T} e^{i x \cdot(u-t)} d x_{1} \ldots d x_{d}= \begin{cases}1, & t=u \in \mathbb{R}^{d}, \\ 0, & t, u \in \mathbb{R}^{d} \text { and } t \neq u .\end{cases}
$$

Denoting the Banach space of $d$-variate almost periodic functions with spectrum within $\boldsymbol{t}$ with respect to the supremum norm by $\mathcal{A} \mathcal{P}_{\boldsymbol{t}}$, one can also identify $\mathcal{F}\left[\mathcal{X}_{\phi}\right]$ with the closure of $\hat{\phi}[\mathcal{A P} \boldsymbol{t}]$ in $L^{2}\left(\mathbb{R}^{d}\right)$. Since $\hat{\phi}\left[L^{\infty}\left(\mathbb{R}^{d}\right)\right]$ is dense in $L^{2}\left(\mathbb{R}^{d}\right)$ and $\mathcal{A P} \boldsymbol{t}$ is not 
dense in $L^{\infty}\left[\mathbb{R}^{d}\right]$, the space $\mathcal{X}_{\phi}$ is a proper closed linear subspace of $H_{\phi}$. Furthermore, due to the estimate

$$
\left\|\sum_{j} c_{j} \kappa_{\phi}\left(\cdot-t_{j}\right)\right\|_{H_{\phi}} \leq\|\phi\|_{2} \sup _{\omega \in \mathbb{R}^{d}}\left|\sum_{j} c_{j} e^{i \omega \cdot t_{j}}\right|,
$$

we see that

$$
\mathcal{A P} \mathcal{P}_{\boldsymbol{t}} \subset \mathcal{A P} \mathcal{t}_{\boldsymbol{t} \phi}=\mathcal{F}\left[\mathcal{X}_{\phi}\right]
$$

When the sampling points form a rectangular grid in $\mathbb{R}^{d}$ containing the origin (i.e., when there exists $\alpha>0$ such that $t_{j}=\alpha j$ for $j \in \mathbb{Z}^{d}$ ), the space $\mathcal{A} \mathcal{P}_{\boldsymbol{t}}$ coincides with the Banach space of all bounded continuous functions $f: \mathbb{R}^{d} \rightarrow \mathbb{C}$ satisfying $f\left(\omega+\frac{2 j \pi}{\alpha}\right)=f(\omega)$ for all $\omega \in \mathbb{R}^{d}$ and $j \in \mathbb{Z}^{d}$, endowed with the supremum norm.

4. Examples. Let us discuss the following examples of real functions. Here we remark that if $\phi(t)$ depends only on $|t|$, then $\kappa_{\phi}(t)$ depends only on $|t|$ and $\hat{\phi}(\omega)$ depends only on $|\omega|$. Consequently, expressing the Cartesian coordinates in spherical coordinates by putting $x_{1}=r \cos \varphi_{1}, x_{2}=r \sin \varphi_{1} \cos \varphi_{2}, \ldots, x_{d-1}=$ $r \sin \varphi_{1} \ldots \sin \varphi_{d-2} \cos \varphi_{d-1}, x_{d}=r \sin \varphi_{1} \ldots \sin \varphi_{d-2} \sin \varphi_{d-1}$, where we have $\varphi_{j} \in$ $[0, \pi](j=1, \ldots, d-2)$ and $\varphi_{d-1} \in[-\pi, \pi]$, with Jacobian

$$
J=r^{d-1}\left(\sin \varphi_{1}\right)^{d-2}\left(\sin \varphi_{2}\right)^{d-3} \cdots \sin \varphi_{d-2},
$$

we obtain

$$
\hat{\phi}(\omega)=(2 \pi)^{-d / 2}\left(\frac{2}{|\omega|}\right)^{\frac{d-2}{2}} \Gamma\left(\frac{d}{2}\right) S_{d-1} \int_{0}^{\infty} r^{d / 2} J_{\frac{d-2}{2}}(|\omega| r) \phi(r) d r,
$$

where $S_{d-1}$ is the surface measure of $S^{d-1}, S_{d-1}=S_{d-2} B\left(\frac{d-1}{2}, \frac{1}{2}\right)$, and $B(p, q)$ and $J_{\nu}(z)$ stand for the Euler beta function and the Bessel function of order $\nu$, respectively.

Example 4.1. A typical example involves the Gram matrix of the multinomial distribution [19]. Let $\Sigma$ be a positive definite real $d \times d$ matrix,

$$
\phi(x)=\left(\frac{\operatorname{det} \Sigma}{\pi^{d}}\right)^{1 / 2} e^{-(\Sigma x, x)}=\pi^{-d / 2}(\operatorname{det} \Sigma)^{1 / 2} \exp \left(-\sum_{i, j=1}^{d} \Sigma_{i j} x_{i} x_{j}\right),
$$

where $x=\left(x_{1}, \ldots, x_{d}\right) \in \mathbb{R}^{d}$ and $\kappa_{\phi}(t, s)=\int_{\mathbb{R}^{d}} \phi(x-t) \phi(x-s) d x$. Then

$$
\kappa_{\phi}(t)=\int_{\mathbb{R}^{d}} \phi(x) \phi(x-t) d x=(2 \pi)^{-d / 2}(\operatorname{det} \Sigma)^{1 / 2} e^{-\frac{1}{2}(\Sigma t, t)},
$$

where $t \in \mathbb{R}^{d}$. In particular, for $t, s \in \mathbb{R}^{d}$ we have

$$
\hat{\phi}(\omega)=(2 \pi)^{-d / 2} e^{-\frac{1}{4}\left(\Sigma^{-1} \omega, \omega\right)} \neq 0
$$

in $\mathbb{R}^{d}$ and

$$
k_{\phi}(t, s)=\int_{\mathbb{R}^{d}} \phi(x-t) \phi(x-s) d x=\kappa_{\phi}(t-s)=\kappa_{\phi}(s-t) .
$$


Hence, if $t_{j}=\alpha j, \alpha>0, j \in \mathbb{Z}^{d}$, the Toeplitz matrix

$$
G_{i-j}(\alpha)=k_{\phi}(\alpha|i-j|),
$$

whose entries have a Gaussian decay away from the diagonal elements, is bounded and strictly positive self-adjoint on $\ell^{2}\left(\mathbb{Z}^{d}\right)$. Furthermore, as $\hat{\phi}$ has no zeros in $\mathbb{R}^{d}$, the expansion (3.7) holds with $\|\phi\|_{2}^{2}=(2 \pi)^{-d / 2}(\operatorname{det} \Sigma)^{1 / 2}$.

Example 4.2. For $\sigma>0$, consider $\phi(x)=e^{-\sigma|x|}$, where the length of $x \in \mathbb{R}^{d}$ is its Euclidean vector norm. Then $\hat{\phi}(\omega)$ depends only on $|\omega|$ and $\kappa_{\phi}(x)$ depends only on $|x|$. For $d=2$ we use $\kappa_{\phi}(t)=k_{\phi}\left(-\frac{1}{2}|t| e_{1}, \frac{1}{2}|t| e_{1}\right)$, where $e_{1}=(1,0)$, and apply the transformation $x=\frac{1}{2}|t|(\cosh u \cos v, \sinh u \sin v)$ to elliptical coordinates $(u, v)$ in (3.1) to find

$$
\kappa_{\phi}(t)=\frac{\pi|t|^{2}}{4} \int_{0}^{\infty} \cosh (2 u) e^{-\sigma|t| \cosh u} d u=\frac{\pi|t|^{2}}{4} K_{2}(\sigma|t|),
$$

where $K_{2}$ stands for McDonald's function [14, 8.432(1)].

For $d \geq 3$ we observe that $(1) \kappa_{\phi}(t)=k_{\phi}\left(-\frac{1}{2}|t| e_{1}, \frac{1}{2}|t| e_{1}\right)$, where $e_{1}=(1,0, \ldots, 0)$, and (2) the integrand does not change if the relative position of $x$ in the twodimensional plane containing $\pm \frac{1}{2}|t|$ and $x$ remains the same. Denoting the surface measure of $S^{d-2}$ by $S_{d-2}$ and using the fact that $S_{d-1}=S_{d-2} B\left(\frac{d-1}{2}, \frac{1}{2}\right)$, we obtain [1, 9.6.23 and 9.6.26]

$$
\begin{aligned}
\kappa_{\phi}(t) & =\frac{S_{d-1}}{2^{d}}|t|^{d} \int_{0}^{\infty}\left[\sinh ^{d} u+\frac{d-1}{d} \sinh ^{d-2} u\right] e^{-\sigma|t| \cosh u} d u \\
& =\frac{\Gamma\left(\frac{d+1}{2}\right) S_{d-1}}{\sqrt{\pi}}\left(\frac{|t|}{2 \sigma}\right)^{d / 2}\left[K_{\frac{d}{2}}(\sigma|t|)+\frac{\sigma|t|}{d} K_{\frac{d-2}{2}}(\sigma|t|)\right] \\
& =\frac{\sigma^{2} \Gamma\left(\frac{d+1}{2}\right) S_{d-1}}{\sqrt{\pi}}\left(\frac{|t|}{2 \sigma}\right)^{\frac{d+2}{2}} K_{\frac{d+2}{2}}(\sigma|t|) .
\end{aligned}
$$

For $d=3$, in particular, we have

$$
\kappa_{\phi}(t)=\frac{\pi}{2}|t|^{3} \int_{1}^{\infty}\left(\xi^{2}-\frac{1}{3}\right) e^{-\sigma|t| \xi} d \xi=\frac{\pi}{\sigma^{3}}\left(\frac{2}{3} \sigma^{2}|t|^{2}+\sigma|t|+1\right) e^{-\sigma|t|} .
$$

Moreover, for any $d \geq 2$ we have

$$
\begin{aligned}
\hat{\phi}(\omega) & =(2 \pi)^{-d / 2} S_{d-2} \int_{0}^{\infty} \int_{0}^{\pi} r^{d-1} \sin ^{d-2} \theta e^{i|\omega| r \cos \theta} e^{-\sigma r} d \theta d r \\
& =(2 \pi)^{-d / 2} S_{d-2} \Gamma\left(\frac{d-1}{2}\right) \Gamma\left(\frac{1}{2}\right)\left(\frac{|\omega|}{2}\right)^{-\frac{d-2}{2}} \int_{0}^{\infty} r^{d / 2} J_{\frac{d-2}{2}}(|\omega| r) e^{-\sigma r} d r \\
& =(2 \pi)^{-d / 2} S_{d-2} B\left(\frac{d-1}{2}, \frac{1}{2}\right) \Gamma(d) \frac{F\left(\frac{d}{2},-\frac{1}{2} ; \frac{d}{2} ; \frac{|\omega|^{2}}{\sigma^{2}+|\omega|^{2}}\right)}{\left(\sigma^{2}+|\omega|^{2}\right)^{d / 2}} \\
& =(2 \pi)^{-d / 2} S_{d-2} B\left(\frac{d-1}{2}, \frac{1}{2}\right) \frac{(d-1) ! \sigma}{\left(\sigma^{2}+|\omega|^{2}\right)^{\frac{d+1}{2}}} \\
& =(2 \pi)^{-d / 2} S_{d-1} \frac{(d-1) ! \sigma}{\left(\sigma^{2}+|\omega|^{2}\right)^{\frac{d+1}{2}}}
\end{aligned}
$$


where we have used (4.1), [14, line 3 of $6.621(1)]$, and $S_{d-1}=S_{d-2} B\left(\frac{d-1}{2}, \frac{1}{2}\right)$, while $F$ stands for the hypergeometric function. For $d=3$ we trivially find

$$
\hat{\phi}(\omega)=\frac{4}{\sqrt{2 \pi}} \frac{\sigma}{\left(\sigma^{2}+|\omega|^{2}\right)^{2}},
$$

which has no zeros in $\mathbb{R}$. As a result, the Gram matrix $G$ given by $G_{i j}=\kappa_{\phi}\left(t_{i}-t_{j}\right)$, $i, j \in \mathbb{Z}^{d}$, whose entries decay exponentially away from the diagonal, is strictly positive definite and the expansion (3.7) holds with $k_{\phi}$ defined as above.

Example 4.3. For $d \geq 2$ and $\sigma>0$ consider the algebraically decaying function $\phi(x)=\left(\sigma^{2}+|x|^{2}\right)^{-\frac{d+1}{2}}$. Then $\phi$ satisfies condition (3.4) for $\gamma \in(d, d+1)$, while (4.1) and $[14,6.565(3)]$ imply

$$
\hat{\phi}(\omega)=(2 \pi)^{-d / 2} \frac{S_{d}}{2 \sigma} e^{-\sigma|\omega|},
$$

where we have employed $S_{d-1} B\left(\frac{d}{2}, \frac{1}{2}\right)=S_{d}$. Thus

$$
\hat{\kappa}_{\phi}(\omega)=(2 \pi)^{d / 2}|\hat{\phi}(\omega)|^{2}=(2 \pi)^{-d / 2} \frac{S_{d}^{2}}{4 \sigma^{2}} e^{-2 \sigma|\omega|} .
$$

Consequently,

$$
\kappa_{\phi}(t)=\frac{S_{d}}{\sigma} \frac{1}{\left(4 \sigma^{2}+|t|^{2}\right)^{\frac{d+1}{2}}} .
$$

More generally, for $d \geq 2, \sigma>0$, and $q=0,1, \ldots$ consider $\phi(x)=\left(\sigma^{2}+|x|^{2}\right)^{-\left(\frac{d+1}{2}+q\right)}$. Then (4.1) and $[14,6.565(4)]$ imply

$$
\begin{aligned}
\hat{\phi}(\omega) & =(2 \pi)^{-d / 2}\left(\frac{|\omega|}{2 \sigma}\right)^{q+\frac{1}{2}} \frac{\Gamma\left(\frac{d}{2}\right) S_{d-1}}{\Gamma\left(\frac{d+1}{2}+q\right)} K_{-\left(q+\frac{1}{2}\right)}(\sigma|\omega|) \\
& =(2 \pi)^{-d / 2} \frac{\theta_{q}(\sigma|\omega|)}{2^{q+1} \sigma^{2 q+1}} \frac{\Gamma\left(\frac{d}{2}\right) \Gamma\left(\frac{1}{2}\right)}{\Gamma\left(\frac{d+1}{2}+q\right)} S_{d-1} e^{-\sigma|\omega|} \\
& =(2 \pi)^{-d / 2} \frac{\theta_{q}(\sigma|\omega|) S_{d}}{2^{q+1} \sigma^{2 q+1}\left(\frac{d+1}{2}\right)_{q}} e^{-\sigma|\omega|},
\end{aligned}
$$

where we have used the Pochhammer symbol $c_{0}=1$ and $c_{s}=c(c+1)(c+2) \cdots(c+s-1)$ for $s=1,2, \ldots$ and the expression (see $[14,8.486(14)$ and 8.486(16)], plus induction on $q$ )

$$
K_{ \pm\left(q+\frac{1}{2}\right)}(z)=\sqrt{\frac{\pi}{2 z}} \frac{\theta_{q}(z)}{z^{q}} e^{-z}
$$

for the so-called Bessel polynomials $\theta_{q}(z)$ of degree $q$ which satisfy the recurrence relations (see $[14,8.486(14)$ and 8.486(10)])

$$
\begin{array}{lll}
\theta_{0}(z)=1, & \theta_{1}(z)=z+1, & \theta_{q+1}(z)=z\left[\theta_{q}(z)-\theta_{q}^{\prime}(z)\right]+(2 q+1) \theta_{q}(z) \\
\theta_{0}(z)=1, & \theta_{1}(z)=z+1, & \theta_{q+1}(z)=(2 q+1) \theta_{q}(z)+z^{2} \theta_{q-1}(z)
\end{array}
$$

and have the explicit form (see $[1,10.2 .15]$ and [15, Chap. 2, (7)-(8)])

$$
\theta_{q}(z)=\sum_{k=0}^{q} \frac{1}{2^{q-k}} \frac{(2 q-k) !}{k !(q-k) !} z^{k}
$$


Using the fact that $\kappa_{\phi}(t)$ is a linear combination of expressions of the type (4.4) with $\sigma$ replaced by $2 \sigma$ as well as (A.1), we obtain

$$
\kappa_{\phi}(t)=\frac{S_{d}}{(2 \sigma)^{2 q+1}[d+1]_{q}} \sum_{s=q}^{2 q} \frac{d_{q, s} \sigma^{2(s-q)}[d+2 q+1]_{s-q}}{\left(4 \sigma^{2}+|t|^{2}\right)^{\frac{d+1}{2}+s}},
$$

where $d_{q, 2 q-n}=\frac{(2 n) !}{2^{n} n !}\left(\begin{array}{l}q \\ n\end{array}\right)(n=0,1, \ldots, q),[c]_{0}=1$, and $[c]_{s}=c(c+2)(c+4) \cdots(c+$ $2 s-2)$ for $s=1,2, \ldots$ In particular, for $q=1$ we find

$$
\kappa_{\phi}(t)=\frac{S_{d}}{8(d+1) \sigma^{3}}\left[\frac{1}{\left(4 \sigma^{2}+|t|^{2}\right)^{\frac{d+3}{2}}}+\frac{\sigma^{2}(d+3)}{\left(4 \sigma^{2}+|t|^{2}\right)^{\frac{d+5}{2}}}\right] .
$$

Further, for $q=2$ and $q=3$ we find

$$
\kappa_{\phi}(t)=\frac{S_{d}}{32 \sigma^{5}(d+1)(d+3)}\left[\frac{3}{\left(4 \sigma^{2}+|t|^{2}\right)^{\frac{d+5}{2}}}+\frac{2(d+5) \sigma^{2}}{\left(4 \sigma^{2}+|t|^{2}\right)^{\frac{d+7}{2}}}+\frac{(d+5)(d+7) \sigma^{4}}{\left(4 \sigma^{2}+|t|^{2}\right)^{\frac{d+9}{2}}}\right]
$$

and

$$
\begin{aligned}
\kappa_{\phi}(t)=\frac{S_{d}}{128 \sigma^{7}(d+1)(d+3)(d+5)}\left[\frac{15}{\left(4 \sigma^{2}+|t|^{2}\right)^{\frac{d+7}{2}}}+\frac{9 \sigma^{2}(d+7)}{\left(4 \sigma^{2}+|t|^{2}\right)^{\frac{d+9}{2}}}\right. \\
+\frac{3 \sigma^{4}(d+7)(d+9)}{\left(4 \sigma^{2}+|t|^{2}\right)^{\frac{d+11}{2}}} \\
\left.+\frac{\sigma^{6}(d+7)(d+9)(d+11)}{\left(4 \sigma^{2}+|t|^{2}\right)^{\frac{d+13}{2}}}\right],
\end{aligned}
$$

respectively. For $q=4$ and $q=5$ we obtain

$$
\begin{aligned}
\kappa_{\phi}(t)= & \frac{S_{d}}{512 \sigma^{9}(d+1)(d+3)(d+5)(d+7)}\left[\frac{105}{\left(4 \sigma^{2}+|t|^{2}\right)^{\frac{d+9}{2}}}\right. \\
& +\frac{60 \sigma^{2}(d+9)}{\left(4 \sigma^{2}+|t|^{2}\right)^{\frac{d+11}{2}}}+\frac{18 \sigma^{4}(d+9)(d+11)}{\left(4 \sigma^{2}+|t|^{2}\right)^{\frac{d+13}{2}}} \\
& +\frac{4 \sigma^{6}(d+9)(d+11)(d+13)}{\left.\left(4 \sigma^{2}+|t|^{2}\right)^{\frac{d+15}{2}}+\frac{\sigma^{8}(d+7)(d+9)(d+11)(d+13)(d+15)}{\left(4 \sigma^{2}+|t|^{2}\right)^{\frac{d+17}{2}}}\right]}
\end{aligned}
$$

and

$$
\begin{aligned}
\kappa_{\phi}(t)= & \frac{S_{d}}{2048 \sigma^{11}(d+1)(d+3)(d+5)(d+7)(d+9)}\left[\frac{945}{\left(4 \sigma^{2}+|t|^{2}\right)^{\frac{d+11}{2}}}\right. \\
& +\frac{525 \sigma^{2}(d+11)}{\left(4 \sigma^{2}+|t|^{2}\right)^{\frac{d+13}{2}}}+\frac{150 \sigma^{4}(d+11)(d+13)}{\left(4 \sigma^{2}+|t|^{2}\right)^{\frac{d+15}{2}}} \\
& +\frac{30 \sigma^{6}(d+11)(d+13)(d+15)}{\left(4 \sigma^{2}+|t|^{2}\right)^{\frac{d+17}{2}}}+\frac{5 \sigma^{8}(d+11)(d+13)(d+15)(d+17)}{\left(4 \sigma^{2}+|t|^{2}\right)^{\frac{d+19}{2}}} \\
& \left.+\frac{\sigma^{10}(d+11)(d+13)(d+15)(d+17)(d+19)}{\left(4 \sigma^{2}+|t|^{2}\right)^{\frac{d+21}{2}}}\right]
\end{aligned}
$$


respectively. In this example the Gram matrix $\left\{k_{\phi}\left(t_{i}, t_{j}\right)\right\}_{i, j \in \mathbb{Z}^{d}}$, whose entries decay algebraically away from the diagonal, is strictly positive self-adjoint. Furthermore, the expansion (3.7) holds with $k_{\phi}$ as above, as $\hat{\phi}$ does not have zeros in $\mathbb{R}^{d}$.

Example 4.4. Now consider the box spline

$$
\phi\left(x_{1}, x_{2}\right)= \begin{cases}1-x_{2}, & 0 \leq x_{1} \leq x_{2} \leq 1 \\ 1-x_{1}, & 0 \leq x_{2} \leq x_{1} \leq 1 \\ 1-x_{1}+x_{2}, & 0 \leq x_{1} \leq 1,-1+x_{1} \leq x_{2} \leq 0 \\ \phi\left(-x_{1},-x_{2}\right), & -1 \leq x_{1} \leq 0,-1 \leq x_{2} \leq 1+x_{1},\end{cases}
$$

and zero elsewhere. Then

$$
\begin{aligned}
\hat{\phi}\left(\omega_{1}, \omega_{2}\right) & =\frac{1}{\pi} \frac{\sin \left(\omega_{1}\right)+\sin \left(\omega_{2}\right)-\sin \left(\omega_{1}+\omega_{2}\right)}{\omega_{1} \omega_{2}\left(\omega_{1}+\omega_{2}\right)} \\
& =\frac{1}{2 \pi} \frac{\sin \left(\frac{1}{2} \omega_{1}\right)}{\frac{1}{2} \omega_{1}} \frac{\sin \left(\frac{1}{2} \omega_{2}\right)}{\frac{1}{2} \omega_{2}} \frac{\sin \left(\frac{1}{2}\left(\omega_{1}+\omega_{2}\right)\right)}{\frac{1}{2}\left(\omega_{1}+\omega_{2}\right)},
\end{aligned}
$$

while $\hat{\phi}(0,0)=(1 / 2 \pi)$ and

$$
\hat{\phi}\left(\omega_{1}, 0\right)=\hat{\phi}\left(0, \omega_{1}\right)=\hat{\phi}\left(\omega_{1},-\omega_{1}\right)=\frac{1}{\pi} \frac{1-\cos \left(\omega_{1}\right)}{\omega_{1}^{2}} .
$$

Thus

$$
\hat{\phi}\left(\omega_{1}, \omega_{2}\right)>0, \quad \max \left(\left|\omega_{1}\right|,\left|\omega_{2}\right|,\left|\omega_{1}+\omega_{2}\right|\right)<2 \pi .
$$

As a consequence, the Gram matrix $\left\{k_{\phi}\left(t_{i}, t_{j}\right)\right\}_{i, j \in \mathbb{Z}^{d}}$ is positive self-adjoint, but the expansion (3.7) is not valid, because $\hat{\phi}\left(\omega_{1}, \omega_{2}\right)$ has zeros in $\mathbb{R}^{d}$.

Let us now employ (3.2) to get

$$
\hat{\kappa}_{\phi}\left(\omega_{1}, \omega_{2}\right)=\frac{4}{\pi} \frac{1-\cos \left(\omega_{1}\right)}{\omega_{1}^{2}} \frac{1-\cos \left(\omega_{2}\right)}{\omega_{2}^{2}} \frac{1-\cos \left(\omega_{1}+\omega_{2}\right)}{\left(\omega_{1}+\omega_{2}\right)^{2}} .
$$

Introducing $\psi(x)=1-|x|$ for $-1 \leq x \leq 1$ and $\psi(x)=0$ for $|x| \geq 1$, so that $\hat{\psi}(\omega)=\sqrt{\frac{2}{\pi}} \frac{1-\cos (\omega)}{\omega^{2}}$, we can write (4.7) in the form

$$
\hat{\kappa}_{\phi}\left(\omega_{1}, \omega_{2}\right)=\sqrt{2 \pi} \hat{\psi}\left(\omega_{1}\right) \hat{\psi}\left(\omega_{2}\right) \hat{\psi}\left(\omega_{1}+\omega_{2}\right),
$$

which implies that

$$
\begin{aligned}
\kappa_{\phi}\left(t_{1}, t_{2}\right) & =\sqrt{2 \pi} \int_{-\infty}^{\infty} d \omega_{1} e^{-i \omega_{1} t_{1}} \hat{\psi}\left(\omega_{1}\right) \int_{-\infty}^{\infty} d z \psi\left(t_{2}-z\right) e^{i \omega_{1} z} \psi(z) \\
& =\int_{-\infty}^{\infty} d z \psi(z) \psi\left(t_{1}-z\right) \psi\left(t_{2}-z\right) \\
& = \begin{cases}S\left(t_{2}\right), & 0 \leq t_{1} \leq t_{2} \leq 2 \\
S\left(t_{1}\right), & 0 \leq t_{2} \leq t_{1} \leq 2 \\
S\left(t_{1}-t_{2}\right), & 0 \leq t_{1} \leq 2,-2+t_{1} \leq t_{2} \leq 0 \\
k_{\phi}\left(-t_{1},-t_{2}\right), & -2 \leq t_{1} \leq 0,-2 \leq t_{2} \leq 2+t_{1},\end{cases}
\end{aligned}
$$


where

$$
S(t)= \begin{cases}\frac{1}{12} t^{3}+\frac{2}{3}(1-t)-\frac{1}{6}(1-t)^{3}, & 0 \leq t \leq 1 \\ \frac{1}{12}(2-t)^{4}, & 1 \leq t \leq 2\end{cases}
$$

and zero outside $[-2,2]^{2}$.

Appendix A. Some expressions involving Bessel polynomials. In this appendix we prove the following result of independent interest.

Theorem A.1. We have

$$
2^{2 q} \theta_{q}(z)^{2}=\sum_{n=0}^{q} \frac{(2 n) !}{2^{n} n !}\left(\begin{array}{c}
q \\
n
\end{array}\right) \theta_{2 q-n}(2 z) .
$$

Proof. According to (5.6) of [8] we have the addition formula

$$
\theta_{q}(z+w)=2^{q} \sum_{r=0}^{q}(-1)^{q-r} \frac{q !(2 r+1)}{(q-r) !(q+r+1) !}(z w)^{q-r} \theta_{r}(z) \theta_{r}(w)
$$

and the inverse addition formula

$$
\theta_{q}(z) \theta_{q}(w)=\sum_{r=0}^{q} \frac{(q+r) !}{(q-r) ! r !} 2^{-r}(z w)^{q-r} \theta_{r}(z+w),
$$

which follow from analogous expressions for the Laguerre polynomials [7]. From (A.3) we have the duplication formula

$$
2^{2 q} \theta_{q}(z)^{2}=\sum_{r=0}^{q} \frac{(q+r) !}{(q-r) ! r !} 2^{r}(2 z)^{2(q-r)} \theta_{r}(2 z) .
$$

Using (3.1) and (1.5) of [8], we see that

$$
z^{2 k} \theta_{n}(z)=\sum_{s=0}^{k}(-1)^{s}\left(\begin{array}{l}
k \\
s
\end{array}\right) \frac{(2 n+2 k+1) ! !}{(2 n+2 k-2 s+1) ! !} \theta_{n+2 k-s}(z),
$$

which generalizes (4.6). Substituting (A.5) into (A.4) (with $2 z, r$, and $q-r$ instead of $z, n$, and $k$ ) we get

$$
\begin{aligned}
2^{2 q} \theta_{q}(z)^{2} & =\sum_{r=0}^{q} \frac{(q+r) !}{(q-r) ! r !} 2^{r} \sum_{s=0}^{q-r}(-1)^{s}\left(\begin{array}{c}
q-r \\
s
\end{array}\right) \frac{(2 q+1) ! !}{(2 q-2 s+1) ! !} \theta_{2 q-r-s}(2 z) \\
& =\sum_{n=0}^{q} \sum_{s=0}^{n}(-1)^{s} 2^{n-s} \frac{(q+n-s) !}{s !(n-s) !(q-n) !} \frac{(2 q+1) ! !}{(2 q-2 s+1) ! !} \theta_{2 q-n}(2 z) \\
& =\sum_{n=0}^{q}\left(\begin{array}{c}
q \\
n
\end{array}\right) B(q, n) \theta_{2 q-n}(2 z),
\end{aligned}
$$


where

$$
\begin{aligned}
B(q, n) & =\sum_{s=0}^{n}(-1)^{s} 2^{n-s}\left(\begin{array}{c}
n \\
s
\end{array}\right) \frac{(q+n-s) !}{q !} \frac{(2 q+1) ! !}{(2 q-2 s+1) ! !} \\
& =2^{n}(n !) \sum_{s=0}^{n} \frac{\left(-q-\frac{1}{2}\right)_{s}(q+1)_{n-s}}{s !(n-s) !}=2^{n}(n !) \frac{\left(\left(-q-\frac{1}{2}\right)+(q+1)\right)_{n}}{n !} \\
& =2^{n}\left(\frac{1}{2}\right)_{n}=\frac{(2 n) !}{2^{n} n !}
\end{aligned}
$$

In the penultimate equality of (A.6) we have applied a corollary of the Chu-Vandermonde identity derived in Remark 2.2.1 of [4].

Appendix B. Condition numbers. In this appendix the condition numbers $\operatorname{cond}\left(G_{\phi}\right)$ of the multi-index Toeplitz matrix $G_{\phi}$ are listed in the case $t_{i}=\alpha i\left(i \in \mathbb{Z}^{d}\right)$ for Examples 4.1-4.4 as far we have been able to compute them, in some cases only for $d=1$.

Example 4.1. For $d=1$ we have $\phi(x)=e^{-\sigma x^{2}}$ and

$$
\begin{aligned}
\hat{G}(s, \alpha) & =\sqrt{\frac{\pi}{2 \sigma}}\left(1+2 \sum_{j=1}^{\infty} e^{-\sigma \alpha^{2} j^{2} / 2} \cos (j \theta)\right) \\
& =\sqrt{\frac{\pi}{2 \sigma}} \vartheta_{3}\left(\frac{1}{2} \theta, e^{-\sigma \alpha^{2} / 2}\right) \\
& =G(\alpha) \sqrt{\frac{\pi}{2 \sigma}} \prod_{j=1}^{\infty}\left\{\left(1+e^{-\left(j-\frac{1}{2}\right) \sigma \alpha^{2}} e^{i \theta}\right)\left(1+e^{-\left(j-\frac{1}{2}\right) \sigma \alpha^{2}} e^{-i \theta}\right)\right\},
\end{aligned}
$$

where $s=e^{i \theta}, \vartheta_{3}$ denotes a Jacobian Theta function [25, sect. 21.11 and 21.3], and $G(\alpha)=\prod_{j=1}^{\infty}\left(1-e^{-j \sigma \alpha^{2}}\right)$. Consequently,

$$
\operatorname{cond}\left(G_{\phi}\right)=\frac{\hat{G}(1, \alpha)}{\hat{G}(-1, \alpha)}=\left(\prod_{j=1}^{\infty} \frac{1+e^{-\left(j-\frac{1}{2}\right) \sigma \alpha^{2}}}{1-e^{-\left(j-\frac{1}{2}\right) \sigma \alpha^{2}}}\right)^{2} .
$$

Example 4.2. For $d=1$ we have $\phi(x)=e^{-\sigma|x|}$ and

$$
\hat{G}(s, \alpha)=\alpha \frac{p(\alpha \sigma)+q(\alpha \sigma)\left[s+s^{-1}\right]}{\left(1-s e^{-\alpha \sigma}\right)^{2}\left(1-s^{-1} e^{-\alpha \sigma}\right)^{2}},
$$

where $p(\beta)=\frac{1}{\beta}-4 e^{-2 \beta}-\frac{1}{\beta} e^{-4 \beta}$ and $q(\beta)=\left(1+\frac{1}{\beta}\right) e^{-3 \beta}+\left(1-\frac{1}{\beta}\right) e^{-\beta}$. Consequently,

$$
\operatorname{cond}\left(G_{\phi}\right)=\frac{\hat{G}(1, \alpha)}{\hat{G}(-1, \alpha)}=\frac{p(\alpha \sigma)+2 q(\alpha \sigma)}{p(\alpha \sigma)-2 q(\alpha \sigma)}\left(\frac{1+e^{-\alpha \sigma}}{1-e^{-\alpha \sigma}}\right)^{4} .
$$

Example 4.3. For $d=1$ and $q=0$ we have $\phi(x)=1 /\left(\sigma^{2}+x^{2}\right)$ and

$$
\begin{aligned}
\hat{G}(s, \alpha) & =\frac{\pi^{2}}{\alpha} \frac{2}{\pi \sigma^{2}}\left(\frac{\alpha}{4 \sigma}+\frac{2 \sigma}{\alpha} \sum_{j=1}^{\infty} \frac{(-1)^{j} \cos \{j(\pi-\theta)\}}{j^{2}+(2 \sigma / \alpha)^{2}}\right) \\
& =\frac{1}{\sigma^{3}} \frac{e^{2(\pi-\theta) \sigma / \alpha}+e^{-2(\pi-\theta) \sigma / \alpha}}{e^{2 \pi \sigma / \alpha}-e^{-2 \pi \sigma / \alpha}},
\end{aligned}
$$


where $s=e^{i \theta}$ (cf. [25, Prob. 9 of Chap. IX]). Consequently,

$$
\operatorname{cond}\left(G_{\phi}\right)=\frac{\hat{G}(1, \alpha)}{\hat{G}(-1, \alpha)}=\cosh \left(\frac{2 \pi \sigma}{\alpha}\right) .
$$

Example 4.4. We now compute the Toeplitz matrix $G=\left(G_{i-j}\right)_{i, j \in \mathbb{Z}^{2}}$ where

$$
G_{i}=\int_{\mathbb{R}^{2}} \phi(x) \phi(x-i) d x, \quad i=\left(i_{1}, i_{2}\right) \in \mathbb{Z}^{2} .
$$

By using (4.8) it is immediate to obtain

$$
G_{i}= \begin{cases}\frac{1}{2}, & i_{1}=i_{2}=0, \\ \frac{1}{12}, & i \in\{(1,0),(-1,0),(0,1),(0,-1)\}, \\ \frac{1}{12}, & i \in\{(1,1),(-1,-1)\}, \\ 0 & \text { elsewhere. }\end{cases}
$$

The corresponding symbol is given by

$$
\hat{G}(s, \alpha=1)=\frac{1}{6}\left(3+\cos \vartheta_{1}+\cos \vartheta_{2}+\cos \left(\vartheta_{1}+\vartheta_{2}\right)\right)>0,
$$

where $s=\left(e^{i \vartheta_{1}}, e^{i \vartheta_{2}}\right)$, from which we immediately have

$$
\operatorname{cond}(G)=\frac{\hat{G}(1,1)}{\hat{G}\left(e^{2 \pi i / 3}, e^{2 \pi i / 3}\right)}=4 .
$$

Acknowledgments. The authors are greatly indebted to Prof. Mourad Ismail for allowing them to conclude the proof of Theorem A.1 by telling them about the $\mathrm{Chu}$-Vandermonde identity in [4]. The authors also express their gratitude to the referees for their useful comments.

\section{REFERENCES}

[1] M. Abramowitz And I. A. Stegun, Handbook of Mathematical Functions, Dover, New York, 1964.

[2] A. Aldroubi And K. GröChEnig, Nonuniform sampling and reconstruction in shift-invariant spaces, SIAM Rev., 43 (2001), pp. 585-620.

[3] D. Alpay, The Schur Algorithm, Reproducing Kernel Spaces and System Theory, SMF/AMS Texts Monogr. 5, AMS, Providence, RI, 2001.

[4] G. E. Andrews, R. Askey, and R. Roy, Special Functions, Encyclopedia Math. Appl. 71, Cambridge University Press, Cambridge, New York, 1999.

[5] N. Aronszajn, Theory of reproducing kernels, Trans. Amer. Math. Soc., 68 (1950), pp. 337404.

[6] H. Bohr, Almost Periodic Functions, Chelsea, New York, 1947.

[7] J. L. Burchnall and T. W. Chaundy, Expansions of Appell's double hypergeometric functions II, Quart. J. Math., Oxford Ser., 12 (1941), pp. 112-128.

[8] L. Carlitz, A note on the Bessel polynomials, Duke Math. J., 24 (1957), pp. 151-162.

[9] T. F. Chan, An optimal circulant preconditioner for Toeplitz systems, SIAM J. Sci. Statist. Comput., 9 (1988), pp. 766-771.

[10] R. J. Duffin and A. C. Schaeffer, A class of nonharmonic Fourier series, Trans. Amer. Math. Soc., 72 (1952), pp. 341-366. 
[11] W. S. ERICKSEn, Inverse pairs of test matrices, ACM Trans. Math. Software, 11 (1985), pp. 302-304.

[12] G. H. Golub And C. F. Van Loan, Matrix Computations, Johns Hopkins University Press, Baltimore, MD, 1983.

[13] T. N. T. Goodman, C. A. Micchelli, and Z. Shen, Riesz bases in subspaces of $L_{2}\left(\mathbb{R}_{+}\right)$, Constr. Approx., 17 (2000), pp. 39-46.

[14] I. S. Gradshteyn and I. M. Ryzhik, Tables of Integrals, Series, and Products, corrected and enlarged ed., Academic Press, New York, 1980.

[15] E. Grosswald, Bessel Polynomials, Lecture Notes in Math. 698, Springer, Berlin, 1978.

[16] P. C. Hansen, Test matrices for regularization methods, SIAM J. Sci. Comput., 16 (1995), pp. 506-512.

[17] E. HiLle, Introduction to general theory of reproducing kernels, Rocky Mountain J. Math., 2 (1972), pp. 321-368.

[18] C. V. M. van Der Mee, M. Z. Nashed, and S. Seatzu, Sampling expansions and interpolation in unitarily translation invariant reproducing kernel Hilbert spaces, Adv. Comput. Math., 19 (2003), pp. 355-372.

[19] C. V. M. van der Mee, G. Rodriguez, and S. Seatzu, Semi-infinite multi-index perturbed block Toeplitz systems, Linear Algebra Appl., 366 (2003), pp. 459-482.

[20] A. Ron And Z. Shen, Frames and stable bases for shift invariant subspaces of $L_{2}\left(\boldsymbol{R}^{d}\right)$, Canad. J. Math., 47 (1995), pp. 1051-1094.

[21] R. SchabaCK, Error estimates and condition numbers for radial basis function interpolation, Adv. Comput. Math., 3 (1995), pp. 251-264.

[22] G. Strang, A proposal for Toeplitz matrix calculations, Stud. Appl. Math., 74 (1986), pp. 171176.

[23] M. Tismenetsky, A decomposition of Toeplitz matrices and optimal circulant preconditioning, Linear Algebra Appl., 154/156 (1991), pp. 105-121.

[24] E. E. TyRTYShnikov, Optimal and superoptimal circulant preconditioners, SIAM J. Matrix Anal. Appl., 13 (1992), pp. 459-473.

[25] E. T. Whittaker and G. N. Watson, A Course of Modern Analysis, 4th ed., Cambridge University Press, London, 1927.

[26] R. M. Young, An Introduction to Nonharmonic Fourier Series, 2nd ed., Academic Press, New York, 2000. 